
\title{
\& Research Square \\ Cryptosporidiosis modulates gut microbiome metabolism and the immune response in an infected host
}

\section{Avinash V Karpe ( $\nabla$ avinash.karpe@csiro.au )}

Commonwealth Scientific and Industrial Research Organisation https://orcid.org/0000-0002-37319598

\section{Melanie L Hutton \\ Monash University}

Steven J Mileto

Monash University

Meagan L James

Monash University

\section{Chris Evans}

Monash University

\section{Rohan M Shah}

Swinburne University of Technology - Hawthorn Campus: Swinburne University of Technology

\section{Amol B Ghodke}

The University of Queensland

\section{Katie E Hillyer}

Commonwealth Scientific and Industrial Research Organisation

\section{Suzanne S Metcalfe}

Commonwealth Scientific and Industrial Research Organisation

Jian-Wei Liu

Commonwealth Scientific and Industrial Research Organisation

\section{Tom Walsh}

CSIRO: Commonwealth Scientific and Industrial Research Organisation

\section{Dena Lyras}

Monash University

\section{Enzo A Palombo}

Swinburne University of Technology - Hawthorn Campus: Swinburne University of Technology

\section{David J Beale}

CSIRO: Commonwealth Scientific and Industrial Research Organisation 
Keywords: Interactomics, host-parasite-microbiome relationships, extra-intestinal effects, D-amino acid/SCFA-induced modulation, Yeast ubiquinone salvation

Posted Date: December 3rd, 2020

DOI: https://doi.org/10.21203/rs.3.rs-119473/v1

License: (c) (1) This work is licensed under a Creative Commons Attribution 4.0 International License. Read Full License 


\section{Abstract \\ Background}

Cryptosporidiosis is a major global human health concern. Despite well-established methods, misdiagnosis remain common. Resulting incorrect clinical prescription often causes anti-microbial resistance development in the patients. Our understanding of the Cryptosporidium infection mechanism remains limited, compounding the difficulty of clinical diagnosis. Multi-omics approach has shown significant potential for addressing this limitation and, biomarker discovery towards rapid and accurate diagnostics. This study investigated the underlying biochemistry of host-microbiome-parasite relationships during infection.

\section{Methods}

C57BL/6J mice were infected with $1 \times 10^{5}$ Cryptosporidium parvum oocysts via oral gavage. Faecal samples were collected daily, while blood, liver tissues and luminal contents of the small and large intestines were collected 10 days post infection. High-resolution liquid chromatography and lowresolution gas chromatography coupled with mass spectrometry were used to analyse the proteomes and metabolomes of faeces, serum, liver, and luminal contents. Faecal samples were additionally subjected to 16S rRNA gene sequencing. Univariate and multivariate statistical analysis were applied to all datasets.

\section{Results}

Host and microbial energy pathways altered during infection. Glycolysis/citrate cycle metabolites, such as malate and lactate, were elevated in the large intestine. Short-chain fatty acids, formate and acetate, increased in the small intestine, while butanoate increased in the caecum-colon. This correlated with an increased abundance of bacteria associated with a stressed host environment, including Lactobacillus (small intestine) and Coriobacteriaceae (throughout the intestine, but more prominently in colon). The expression of host electron transfer flavoprotein, phosphoglycerate kinase and acetyl CoA binding proteins, yeast glyceraldehyde-3-phosphate dehydrogenase, and Lactobacillus glyceraldehyde-3phosphate dehydrogenase significantly increased in the infected gut. Liver oxalate increase was also seen during infection.

\section{Conclusions}

The microbiome-parasite relationship is more influential than the previously thought host-parasite relationship, in mediating major biochemical changes in the mouse gut during cryptosporidiosis. Defining this parasite-microbiome interaction is the first step towards building a comprehensive cryptosporidiosis model. 


\section{Background}

Enteric protozoal infections are a major human health concern globally, causing malnutrition through the loss of appetite, decreased nutrient absorption, and increased catabolism of nutrient reserves due to inflammation and diarrhoea. Cryptosporidiosis is a globally endemic infection with about 4.7 million annual reported cases [1] with high impact on children aged 4 years or below and immunocompromised individuals [2]. The infection is caused by Cryptosporidium parvum (henceforth indicated as Cryptosporidium), a major waterborne enteric pathogen [1]. Cryptosporidium is a highly specialised obligate apicomplexan parasite that is transmitted via the faecal-oral route from sources such as drinking water, or recreational waters contaminated with sewage and/or animal faeces [3]. Due to its ability to infect humans and other mammals, it is considered a ubiquitous zoonotic parasite [2, 3].

Cryptosporidium infections are limited to the epithelial lining of the gastrointestinal tract, causing minimal invasion and penetration through mucosal layers, and are known to be autophagic [4]. Due to its highly specialised lifecycle, Cryptosporidium lacks numerous metabolic systems and must interact with its host to compensate for these deficiencies [5-7]. This significant intertwined relationship between a host and Cryptosporidium is, therefore, highly complex but is only partially understood. It has been shown that cryptosporidiosis causes long-term pan-body effects such as weight loss, abdominal, eye, and joint pain and, very likely, irritable bowel syndrome (IBS) [8]. However, the molecular mechanisms and host responses that result in these broad disease effects are not well understood.

In this context, a multi-omics approach will provide broader systems biology information relating to cryptosporidiosis. Omics platforms such as genomics, proteomics, and metabolomics, alone or in combination, have provided new insights that have been valuable in preventative health $[9,10]$, toxicology, and medicine $[11,12]$. The high sensitivities and specificities of multi-omics platforms provide excellent discrimination between samples and treatment types and have been applied to study environmental, clinical, and natural medicine systems [13-15]. Metabolomics and genomics have been independently applied to understand the Cryptosporidium life cycle in aquatic systems [16, 17], and invitro studies $[4,6,7]$. Metabolomics or gut microbial community genomics studies have been performed separately on the infected host $[18,19]$. Furthermore, a multi-omics approach will provide broader systems biology information relating to cryptosporidiosis effects and host reponses.

In this study, we investigated interactions between $C$. parvum and a murine host during cryptosporidiosis using multi-omic platforms (metabolomics and proteomics). To define the host-specific interactions of $C$. parvum, infection with the bacterium uropathogenic Escherichia coli (UPEC) [20], and the eukaryotic pathogen Giardia lamblia was used for comparative purposes. Gut infection with UPEC does not appear to cause disease symptoms in humans or mice [21], while Giardiasis results in similar symptoms to those seen in cryptosporidiosis [2]. Untargeted metabolomics, proteomics, and microbiome 16S rRNA gene sequencing were applied to numerous body tissues and gut washes after infection with $C$. parvum or abovementioned infectious agents. We examined how cryptosporidiosis modulates the enteric microbial community profile and, affects host protein levels and metabolic processes throughout the mouse 
intestinal tract and in extra-intestinal tissues. This work also provided new insights into previously unreported dynamics of microbiome, parasite and host relationships in different regions of the gastrointestinal tract during cryptosporidiosis.

\section{Methods}

\section{Animal Ethics and husbandry}

All experiments were approved by the Monash University Animal Ethics Committee (Monash University AEC no. MARP/2018/055) following the guidelines of Victorian State Government and The National Health and Medical Research Council, Australian Government. Mice were housed in Optimice cages containing sterile sawdust at $18-24^{\circ} \mathrm{C}, 40-70 \%$ humidity, and 12:12 hour light/dark cycle. Mice were provided with sterile water and feed (Ridely AgriProducts Pty. Ltd., Melbourne, VIC, Australia) ad libitum.

\section{Mouse infection model}

For the study, groups of five, three-week-old C57BL/6J female mice were acclimatised for one week before infection with either $1 \times 10^{5} \mathrm{C}$. parvum or G. lamblia oocysts (C. parvum, Cat. Number: C10E7; G. lamblia, cat. Number: G10E6; BTF Pty Ltd., North Ryde, NSW, Australia) or $1 \times 10^{8}$ CFU of UPEC (ST131 lineage strain EC958) via oral gavage. An additional group of uninfected mice $(n=5)$ was also included for comparison against the infected groups. Cryptosporidium and Giardia infection was monitored for 10 days via daily faecal collection and detection of oocysts by fluorescent microscopy using the EasyStain kit $^{\text {TM }}$ (Biopoint Pty Ltd., Sydney, Australia). UPEC infection was quantified using a previously described method [20]. Mice were euthanised at 10 days post-infection by $\mathrm{CO}_{2}$ exposure and liver tissue, serum, and faeces collected. The luminal contents of the duodenum, jejunum, ileum, caecum, and colon were sampled by flushing $1.0 \mathrm{~mL}$ of sterile phosphate buffer saline through each section of the gut and collecting the contents (Figure 1). Serum (collected by cardiac bleed) and liver tissue samples were used as representatives for indirect and cross-organ effects of $C$. parvum infection. The luminal contents represented the direct effects of infection. All samples were immediately stored on dry ice and then at $-80^{\circ} \mathrm{C}$ until further analysis.

\section{Untargeted metabolomics by gas chromatography-mass spectrometry (GC-MS)}

For untargeted metabolomics analysis, samples were prepared as previously described [22], with minor modifications. Briefly, frozen samples of faecal pellets (10 - 25 mg, wet weight), serum (10 - 15 mg, wet weight), liver (40 - 50 mg, wet weight) and, duodenum, jejunum, ileum, caecum, and colon washes (200 $250 \mathrm{mg}$, wet weight) were transferred to $1.5 \mathrm{~mL}$ homogenisation tubes (Navy RINO lysis kit, BioTools Pty. Ltd., Keperra, QLD, Australia). A $1 \mathrm{~mL}$ aliquot of chilled extraction solution $\left(-20^{\circ} \mathrm{C}\right)$ comprising acetonitrile, isopropanol, and water $(3: 3: 2, \mathrm{~V} / \mathrm{V} / \mathrm{V})$ spiked with 'Internal Standard $1^{\prime}$ (Valine ${ }^{13} \mathrm{C}_{2}$ and Stearic acid ${ }^{13} \mathrm{C}$, both $10 \mu \mathrm{g}$; Novachem Pty. Ltd., Heidelberg West, VIC, Australia) was then added to each of the sample tubes. The samples were then homogenised at 6,800 rpm for $2 \times 20$ sec cycles, with 10 sec rest, at room 
temperature (Percelleys Evolution, Bertin Instruments, Montigny-le-Bretonneux, France). The homogenised samples were centrifuged at $14,000 \mathrm{~g}$ for $2 \mathrm{~min}$ at $4^{\circ} \mathrm{C}$. A $100 \mu \mathrm{L}$ aliquot of the supernatant was transferred to a glass vial with fused inserts. The samples were dried in a vacuum centrifuge at $37^{\circ} \mathrm{C}$. Upon drying, $50 \mu \mathrm{L}$ Myristic acid- $\mathrm{d}_{27}$ (Sigma Aldrich; $0.2 \mathrm{mg} / \mathrm{mL}$ in methanol) was added as 'Internal Standard 2'. The samples were re-dried in a vacuum centrifuge. The samples were derivatised 'in-time', followed by a 1 -hour holding time, before injection into a GC-MS as previously reported $[23,24]$.

\section{Metaproteome extraction and proteome analysis}

Proteomics samples comprised serum and liver tissues (wet weight $=50 \pm 2.5 \mathrm{mg}$ ), $250 \mathrm{mg}$ of luminal contents (duodenum, jejunum, ileum, caecum, and colon), and faeces $(20 \mathrm{mg})$. Samples were weighed and transferred to $1.5 \mathrm{~mL}$ bead mill homogeniser tubes. Urea (8M, in $20 \mathrm{mM}$ Tris, $\mathrm{pH} 8,50 \mu \mathrm{L})$ was added, except for luminal content samples. Samples were homogenised at a frequency of $28 \mathrm{~s}^{-1}$ for $3 \times 10 \mathrm{~min}$ in a Qiagen TissueLyzerll system (Qiagen Pty Ltd., Chadstone, VIC, Australia). Samples were centrifuged $\left(16,000 \mathrm{~g}, 5 \mathrm{~min}, 4^{\circ} \mathrm{C}\right)$, and the supernatant transferred to new tubes. Room temperature MilliQ water (50 $\mu \mathrm{L})$ was added, followed by cold acetone $\left(-20^{\circ} \mathrm{C}, 400 \mu \mathrm{L}\right)$. The samples were incubated at $-20^{\circ} \mathrm{C}$ for $1 \mathrm{~h}$ and centrifuged $\left(16,000 \mathrm{~g}, 5 \mathrm{~min}, 21^{\circ} \mathrm{C}\right)$. The supernatant was decanted, and the pellet was re-washed with $200 \mu \mathrm{L}$ acetone, followed by re-centrifuging and air-drying. Samples were incubated in $8 \mathrm{M}$ urea for 1 h, followed by water bath sonication (room temperature, $10 \mathrm{~min}$ ) and centrifugation $(16,000 \mathrm{~g}, 5 \mathrm{~min}$, $21^{\circ} \mathrm{C}$ ). The supernatant was transferred to a fresh tube.

The protein concentration of the cleaned extract was determined by Bradford assay and a volume equivalent to $5 \mu \mathrm{g}$ of protein was taken. The volume was adjusted to $10 \mu \mathrm{L}$ by adding urea $(8 \mathrm{M})$. Dithiothreitol $(1 \mu \mathrm{L}, 15 \% \mathrm{~V} / \mathrm{v})$ and acrylamide $(1 \mu \mathrm{L}, 40 \% \mathrm{v} / \mathrm{v})$ were added, each followed by a 30-min incubation $\left(25^{\circ} \mathrm{C}\right)$. After trypsin $(0.1 \mu \mathrm{g}$ in $20 \mathrm{mM}$ ammonium bicarbonate, $47 \mu \mathrm{L})$ was added, the mixture was incubated for $3 \mathrm{~h}$ at $37^{\circ} \mathrm{C}$. Formic acid $(1 \mu \mathrm{L}, 10 \% \mathrm{v} / \mathrm{v})$ was added to terminate trypsin activity.

Tryptic peptides (100 ng) were desalted and concentrated with a trap column (PepMap100 C18 $5 \mathrm{~mm} \times$ $300 \mu \mathrm{m}, 5 \mu \mathrm{m}$ ) and separated on a nano column (PepMap100 C18 $150 \mathrm{~mm} \times 75 \mu \mathrm{m}, 2 \mu \mathrm{m})$ using an Ultimate $^{\mathrm{TM}} 3000 \mathrm{RSLC}$ nano-LC system, with mobile phases (A: water $+0.1 \%(v / v)$ formic acid; $\mathrm{B}$ : acetonitrile $(80 \% \mathrm{v} / \mathrm{v})+0.08 \%(\mathrm{v} / \mathrm{v})$ formic acid). The peptides were eluted using Solvent $\mathrm{B}$ at gradient $\mathrm{s}$ of 5 - 40\% (0 - $60 \mathrm{~min}$ ) and 40 - 99\% (60 - $70 \mathrm{~min}$ ). The eluted peptides were ionized with a Nanospray Flex lon Source (Note: All instruments and parts of Liquid chromatography-High resolution mass spectrometry (LC-HR-MS) were sourced from Thermo Scientific Australia Pty Ltd, Scoresby, VIC, Australia). Protein Discoverer 2.2 (Thermo Scientific) and Sequest HT search engine were used to identify peptides/proteins and quantify the relative abundance of proteins (Further details are provided in the supplementary section).

\section{Genomic extraction, analysis, and processing}

Mouse faeces and luminal contents ( $n=5$ each) were homogenised and DNA was extracted using the manufacturer's instructions (ZymoBiomics DNA miniprep kit, Zymo Research Corp., Irvin, CA, USA), 
followed by DNA amplification, sequencing, and analysis (Supplementary Materials). Sequence analysis was performed using QIIME 2 (Release no. 2019.7) pipeline [25], as previously described [15]. Multivariate statistics using METAGENassist analysis [26] were performed to investigate the metabolic nature of the microbial community detected in each sample group.

\section{Multi-omics integration and statistical analysis}

The metabolomics and proteomics data were adjusted for batch-effect, log transformed and multivariate data analysis conducted with the software SIMCA (version 16, Sartorius Stedim Biotech, Umeå, Sweden) and MetaboAnalyst 4.0 [27]. The cut-off level for significant metabolites was a signal-to-noise $(S / M)$ ratio of 10 , while for proteins, it was a relative abundance of $1 \times 10^{5}$. For statistical analysis of both metabolome and proteome, a fold change of $\leq 0.5$ (downregulation) or $\geq 2.0$ (upregulation), and a Benjamini-Hochberg adjusted $p$-value of $\leq 0.05$. Metabolic and proteomic outputs were integrated using the 'Joint-pathway analysis tool' of Metaboanalyst 4.0 and Paintomics 3[28].

The metabolic pathway networks obtained after statistical analyses were manually curated in Omix visualization software (Version 1.9.34; Omix Visualisation GmbH and Co. KG, Lennestadt, Germany).

\section{Results}

\section{Microbiome distribution and, protein and metabolic expression in the gut}

Interaction dynamics in the infected host's gastrointestinal tract during cryptosporidiosis were assessed by (i) the response of the host's system and gut microbiome, and (ii) the effects on non-gut organs, as detailed below.

The genomic analysis of luminal contents and faeces performed via rarefaction analysis and Good's coverage index (Table S1) indicated good sequencing depth (Figure S2A) towards representational operational taxonomic units (OTUs). The sequencing analysis indicated 847 features detected in all samples, represented by $65.39 \%$ retained sequences. The average feature count of the uninfected group was 24,188 and 24,290 for samples infected with Cryptosporidium.. The sequencing efficiency was determined by comparing the percentage of different OTUs identified in the microbial community standard II sample (Log distribution) (ZymoBiomics D6310, Zymoresearch Corp., Irvine, CA, USA) with the manufacturer's data. Of the 8 species in the standard sample, 7 were detected, representing the expected proportion. The remaining species in the standard, Staphylococcus aureus, was not detected. This was most likely due to an extremely low percentage of genomic DNA composition $(0.000089 \%)$ within the standard sample. Proteomic analysis of luminal contents and faeces of Cryptosporidium-infected mice indicated 4,239 proteins expressed by the host, with a good fit $\left(R^{2} X=0.88, R^{2} Y=0.799\right)$ and predictability $\left(Q^{2}=0.41\right)$ (Figures S3B and S3C). Searches against 43 microbial UniProt databases showed that the number of expressed microbiome proteins increased from the duodenum (30 proteins) through to colon (815 proteins) and faeces (956) (Figure S3A). The metabolome output showed the presence of 162 metabolites across all the analysed samples (Figure S4, Table S2Table S3). 


\section{Gut microbiome response during cryptosporidiosis}

The 16S rRNA gene analysis by Greengenes database assigned OTUs to 71 bacterial genera. Of these, 22 genera were represented in all luminal contents and faeces and were indicated as the core microbial community (Figure 2A). Whilst Faecalibaculum, Barnesiella, and Lactobacillus were abundant in the small intestine, the Ruminococcaceae population increased in the caecum and colon (Figure 2B). During cryptosporidiosis, in the small intestine, beneficial bacteria such as Faecalibaculum and Lachnospiraceae showed considerable depletion, while Lactobacillus, Lachnospiraceae, Desulfovibrio, and Coriobacteria populations increased (Figure 2B).

The role of the microbiome in the production of short-chain fatty acid (SCFAs) in the gut [29] is known, especially during induced gut disorder stress [15]. We examined whether cryptosporidiosis-induced changes in the microbiota composition affected SCFA production in the gut of infected mice. We observed that among SCFAs, formate had a higher metabolic expression in the small intestine (duodenum > jejunum > ileum), while acetate, propanoate, and butanoate accumulation increased in the caecum and colon during cryptosporidiosis (Figure $3 \mathrm{~A}$ ). In addition to SCFAs, the accumulation of Damino acids, such as D-alanine and D-proline, in the small intestine (Figure 3B) reflected an increased abundance of Lactobacillus (Figures 2B), supporting the observations of Sasabe et al [30].

An increase in protective/stress response (microbiome) proteins with the increase in Lactobacillus (or similar bacterial) population was also indicated by the increased levels of proteins responsible for glycolysis and fatty acid metabolism (Figure 4A). The upregulated proteins (Note: all Uniprot IDs. Individual database IDs are provided in Supplementary materials section) in the duodenum consisted of Enterococcus dicarboxylate transporter (Q820V1, FC $=1.41$ ) and histone $\mathrm{H} 4$ proteins of Candida (C5M3N6, FC $=1.15)$, Saccharomyces (P02309, FC $=1.15)$ and Cryptosporidium (Q5CV68, FC = 1.15). To analyse protein expression across the intestine, data from different sections of the small intestine were combined in the Biomarker meta-analysis tool of Metaboanalyst 4.0. The output was obtained as combined LogFold change (cFC) with the minimum cut-off of $\mathrm{cFC}=1$ and $p$-value (FDR adjusted) $\leq 0.05$. During cryptosporidiosis, compared to other regions of intestine, proteins significantly upregulated in the jejunum-ileum included glyceraldehyde-3-phosphate dehydrogenases from yeasts ( $P 00360, \mathrm{cFC}=11.02$ ) and Lactobacillus (A0A062X383, CFC = 1.41) (Figure 4A, Supporting dataset 1).

The caecum and colon showed the expression of different prokaryotic proteins. The expressed proteins mainly related to the glycolysis pathway, leading to fatty acid synthesis and oxidative stress protection proteins such as rubrerythrins (Figure 4B).

\section{Host-response in the gut during cryptosporidiosis}

The activation of host defence systems showed a considerable increase in the jejunum where numerous immune-type proteins were expressed during infection. Host response proteins associated with protective and inflammatory responses (actins, myosins, keratins, heat shock proteins, apoptosis-associated 
proteins), oxidative stress (glutathione S-transferase, GTPases, selenium binding proteins), and glycolysis/gluconeogenesis-associated enzymes were observed (Table S9, Supporting dataset 2). Immunity-related heat shock proteins, namely actins and tubulins, were expressed along with glycolysisrelated enzymes such as glyceraldehyde-3-P dehydrogenase and dihydrolipoyl dehydrogenase. Cryptosporidium proteins such as actin $(F C=3065.1)$, tubulin $(F C=1040.6)$, and heat shock proteins (FC of HSP90 = 2483; HSP $70=197.2$ ) showed considerable upregulation in the ileum, indicative of increased expression during infection. Additionally, 3064 host response proteins were expressed in the ileum, the highest among all the intestinal regions (Figure S3).

During cryptosporidiosis, most metabolites depleted in the small intestine. Primary decreased metabolites in the duodenum (Figure 5A, Table S3) included succinate, 6-hydroxy caproate, adenosine and inosine monophosphates, and glucose. In the jejunum, maleate, non-hexose sugars, and sugar acids (glucoheptonate and sedoheptulose) were decreased (Figure 5A, Table S4). In the ileum, depleted metabolites included sorbose, erythrose phosphate, ribitol, glycerol, and gluconate. Metabolites such as shikimate, phenaceturate, and urea were elevated (Figure 5A, Table S5). Overall, during cryptosporidiosis, more metabolites, especially sugars, sugar acids, and sugar alcohols, were decreased in the small intestine than upregulated.

On the contrary, the number of elevated metabolites increased in the caecum and colon during infection. In the caecum, amino acids such as glycine, methionine, creatinine, tyrosine, alanine, lysine, and cysteine were increased (Figure 5A, Table S6). Other increased metabolites in the colon included fatty and organic acids, such as malate, 3-aminoisobutyrate, fumarate, 3,4-dihydroxymandelate, and citrate (Figure 5A, Table S7). The metabolic composition of the faeces was similar to that of the colon, with addition of increased abundances of organic acids and non-digestible sugars, such as cellobiose (Figure 5A, Table S8). Overall, metabolic activity, combined with protein expression, indicated that Cryptosporidium metabolic activity was most prominent in the small intestine, followed by a decline in the caecum and a spike in the colon and faeces.

The integrated joint-pathway analysis of metabolic-proteomic datasets showed 69 key metabolic pathways being expressed, of which 10 were statistically significant with respect to uninfected mice (Holm adjusted p-value $\leq 0.05$ ) (Table 1 ).

Table 1. Most significant metabolic pathways in the gut modulated during cryptosporidiosis with respect to the uninfected mice, based on integration of the metabolomics-proteomics data using a joint pathway analysis tool. 


\begin{tabular}{|c|c|c|c|c|}
\hline Metabolic pathway & Match status & Impact & $\begin{array}{l}\text { P-value } \\
\text { (Holm adj. }\end{array}$ & FDR \\
\hline Arginine biosynthesis & $13 / 27$ & 1.12 & $2.37 e^{-07}$ & $2.37 \mathrm{e}^{-07}$ \\
\hline Citrate cycle (TCA cycle) & $15 / 42$ & 1.95 & $2.02 \mathrm{e}^{-06}$ & $1.02 \mathrm{e}^{-06}$ \\
\hline Glycolysis or Gluconeogenesis & $16 / 61$ & 1.28 & $8.69 e^{-05}$ & $2.97 \mathrm{e}^{-05}$ \\
\hline Pyruvate metabolism & $13 / 45$ & 0.93 & 0.0003 & $7.47 e^{-05}$ \\
\hline Nitrogen metabolism & $6 / 10$ & 1.00 & 0.0011 & 0.0002 \\
\hline Glutathione metabolism & $13 / 56$ & 0.69 & 0.0038 & 0.0006 \\
\hline Alanine, aspartate and glutamate metabolism & $13 / 61$ & 0.83 & 0.0097 & 0.0015 \\
\hline Glyoxylate and dicarboxylate metabolism & $12 / 56$ & 0.53 & 0.0167 & 0.0023 \\
\hline Galactose metabolism & $11 / 51$ & 0.66 & 0.0287 & 0.0035 \\
\hline Arginine and proline metabolism & $14 / 78$ & 0.52 & 0.0349 & 0.0039 \\
\hline
\end{tabular}

Note: Match status = number of (significant metabolites and proteins/total metabolites and proteins) in a pathway; $F D R=$ false discovery rate

\section{Proxy-citrate cycle and fatty acid metabolism}

It appeared that during infection, citrate, succinate, oxalate, malate, glycolate, and orthophosphate were catabolised more in the small intestine than the large intestine (Figure 5A, 6, and S7). Proteins related to the citrate cycle and oxidative phosphorylation were expressed across the mouse intestine during cryptosporidiosis (Figure 5B). The regression analysis indicated increased catabolism of orthophosphate throughout the intestinal tract (Figure 5A), except in faeces. Among proteins, the highest expressions (cFC $>2$ ) were related to oxidative phosphorylation and glycolysis (Table S9). Other energy generation pathways such as glutamate metabolism possibly assisted Cryptosporidium to create a proxy-citrate cycle. These involved host mitochondrial NADH dehydrogenases [ubiquinone] (Uniprot IDs: D3YUK4, Q99LY9, Q9Z1P6, and Q9D6J6; cFC = 1.35). These results indicate that considerable oxidative phosphorylation is necessary to maintain highly upregulated citrate cycle activities (Figure 6) during cryptosporidiosis [5].

In the ileum, Saccharomyces mitochondrial dihydrolipoyl dehydrogenase (P09624; FC $=83.08$ ) and Candida malate dehydrogenases (C5M2D7, FC $=817.11$ and Q5AMP4, FC $=547.99)$ were highly upregulated. Here, localised glutamine synthetase upregulation (P26443, FC $=2.33$ ) and overall glutamate dehydrogenase downregulation (F7CFA5, $\mathrm{CFC}=-0.94$ ) indicated reduced glutamate utilisation by the host, and its likely salvaging by Cryptosporidium (Figure 6). 
Changes in fatty acid metabolism, especially medium-chain and long-chain fatty acids (MCFAs and LCFAs), were observed in the intestine upon Cryptosporidium infection. Although fatty acid oxidation and glycerolipid metabolism was observed in the duodenum, the latter was more prominent throughout the small intestine, as indicated by a significant decrease of glycerol in the jejunum ( $F C=0.07)$ and the ileum $(F C=0.04)$, and palmitate $(F C=0.26)$ and palmitoleate $(F C=0.22)$ in the ileum.

\section{Proxy-citrate cycle protein expression during cryptosporidiosis compared to G. lamblia and UPEC infection}

To ascertain if the protein profile observed in the gut was specific to cryptosporidiosis, we compared the proteomic output during cryptosporidiosis to that obtained from a UPEC gut infection or Giardia infection. Immune precursor proteins such as actins showed similar expression across the intestine (cFC $1-6.2$ ) across all three infections. However, proteins related to oxidative phosphorylation and glycolysis had higher expression during cryptosporidiosis when compared to UPEC infection and Giardiasis (Table S9). Additionally, some proteins with high expression during cryptosporidiosis such as ADP/ATP translocase ( $\mathrm{cFC}=3.59)$, electron transfer flavoproteins ( $\mathrm{CFC}=2.5-2.63$ ) and acyl $\mathrm{CoA}$ binding proteins $(\mathrm{cFC}=2.08)$ were either non-significantly ( $p$-value $\geq 0.05$ ) different or were downregulated ( $p$-value $\leq 0.05$ ) during Giardia or UPEC infection. Also, related proteins such as glutathione peroxidase ( $\mathrm{cFC}=2.38$ ) and phosphoglycerate kinase $(\mathrm{cFC}=2.07$ ) had significantly higher expression during cryptosporidiosis (Table S9). The analysis indicated that the proxy-citrate cycle was specifically upregulated during cryptosporidiosis concerning other gut infections.

\section{Ubiquinone biosynthesis (coenzyme Q) pathway salvaging}

During cryptosporidiosis, metabolites from the pentose phosphate pathway (PPP) were downregulated in the small intestine, particularly in the ileum. These included sedoheptulose $\left(F C=4.74 \mathrm{e}^{-05}\right)$, erythrose-4phosphate $(\mathrm{E} 4 \mathrm{P})(\mathrm{FC}=0.0004)$, ribitol $(\mathrm{FC}=0.02)$, sorbose, tagatose and glucoheptonate $($ all, $\mathrm{FC}=$ 0.0001). On the other hand, shikimate was considerably upregulated in the ileum ( $F C=32.65)$. This activity indicated aromatic amino acid biosynthesis via the E4P " shikimate " chorismate route. In the current study, this pathway appeared to be expressed through salvaging of host transketolase (P40142), primarily in the jejunum $(F C=2.08)$ and ileum $(F C=1.70)$ and yeast transaldolase $(F C=2.1)$, followed by yeast polyubiquitin proteins in the ileum $(F C=1107)$. Additionally, the joint-pathway analysis also indicated that the role of host ubiquinone protein expression towards ubiquinone biosynthesis in the gut was limited (Impact $=0.06$, p-value $=0.6918)$. On the other hand, the yeasts played a major role in ubiquinone biosynthesis (Figure 6), as indicated by statistically significant tyrosine metabolism (Impact = $0.57, \mathrm{p}$-value $=0.0258)$, phenylalanine, tyrosine and tryptophan biosynthesis $(\operatorname{Impact}=1.4, \mathrm{p}$-value $=$ 0.0323 ), and ubiquinone and other terpenoid-quinone biosynthesis (Impact $=0.5, p$-value $=0.1$ ) in the ileum.

\section{Extra-intestinal effects of cryptosporidiosis}


Few studies have focused on the effects of enteric infection on non-gut organs and, to our knowledge, no studies have addressed this for cryptosporidiosis. For this study, serum and liver were used as representative samples for measuring extra-intestinal effects, such as nutrient absorption, detoxification, and immune response.

During cryptosporidiosis, we observed downregulation of fatty acid metabolism in the serum; the major fatty acids affected were palmitoleate $(F C=0.07)$, oleate $(0.05)$, and myristate $(0.02)$ when compared to the uninfected mice (Table S10). In the liver, we observed a similar decrease, specifically of 6-hydroxy caproic acid and succinic acid (Table S11).

During cryptosporidiosis, of 1320 and 3016 expressed proteins in serum and the liver, respectively, 327 were significantly upregulated across both (Supporting Data 2). These included immune response proteins, such as myosins and selenium binding proteins. Complement factors $\mathrm{H}(\mathrm{cFC}=3.97$ ) and $\mathrm{B}$ ( $\mathrm{cFC}$ $=3.2-3.55)$, immunoglobulins ( $\mathrm{CFC}=2.04-2.76$ ) and apolipoproteins $(\mathrm{CFC}=1.87-3.03$ ) showed statistically significant expression when compared to the uninfected mice. Additionally, the metabolismrelated proteins important for gluconeogenesis, Krebs cycle, and phosphorylation, such as mitochondrial pyruvate carboxylase and creatine kinase (M-type), were highly expressed during infection (Table S12, Supporting dataset 3$)$.

\section{Discussion}

\section{Cryptosporidiosis dynamics in the gut}

The microbiome generally forms the first line of defence upon the onset of parasite infection. Numerous mechanisms play a role in the Cryptosporidium-microbiome relationship during cryptosporidiosis that can be used to further elaborate on the dynamics of this zoonotic infection in humans [31]. Considered among the core microbiome population, Faecalibaculum promotes higher SCFA metabolism, maintaining ionic balance and controlling virulence factors secreted by bacterial pathogens [32]. Synthesis and metabolism of SCFAs by the gut microbiome modulate inflammatory cytokine activity [33], especially by increased butanoate and propionate production in the caecum and colon [29]. Among the gut microbial community, Faecalibaculum and members of the Erysipelotrichaceae are known to produce high levels of lactate and formate [34,35]. In the current study, formate showed considerable contribution towards both pyruvate and glycolysis metabolism (Figures 2A and S5, and S7). A depletion of Faecalibaculum (Figure 2) due to Cryptosporidium infection in this study would, therefore, explain the microbiome dysbiosis and decreased pyruvate and glycolysis metabolism in the small intestine.

An increased Desulfovibrio population, on the other hand, has been shown to perturb microbiota during protozoal gut infections. Desulfovibrio is known to cause enteritis through mucosal or epithelial damage by releasing toxic compounds such as hydrogen sulphide in the gut environment [36]. We observed that bacteria such as Coriobacteriaceae and Lactobacillus, increased in abundance during Cryptosporidium infection, especially in the small intestine. Coriobacteriaceae have been demonstrated to modulate glucose metabolism [37]. Similarly, the increased level of Lactobacillus may represent a microbial 
response for countering the mucosal/epithelial damage caused by Cryptosporidium infection. Reportedly, lactate metabolising bacteria are highly active in the production of D-amino acids [38] and, provide an elevated microbial response to balance the mucosal/epithelial damage caused by Cryptosporidium infection [36].

Microbial carboxylase transporters are part of tripartite ATP-independent periplasmic (TRAP) and Tripartite Tricarboxylate Transporters (TTT) protein families. These proteins induce pathogenicity and colonisation of bacteria such as Haemophilusinfluenzae and Salmonella Typhimurium. This activity uses energy sources, such as glutamate, and causes increased levels of dicarboxylic acids, for instance, acetate or hexanoate (Figure 3A) [39]. Cryptosporidium excystation in the duodenum has been documented [3] and may be responsible for the observed upregulation of proteins assoaciated with glycolysis, glutaminolysis, and citrate cycle in the small intestine. We found that the citrate cycle was more active across the intestine during cryptosporidiosis (Figure 5A, Figure 3A, 4A, 6, and S7). C. parvum reportedly lacks the machinery for the citrate cycle pathway and salvages it from the host [40]. However, the current study shows, for the first time, a greater role of yeasts in driving this proxy-citrate pathway. Additionally, the pathway may have also contributed to fatty acid metabolism. In particular, malonate catabolism ( $F C=0.35)$ and fatty acid synthase activity (AOAOU1RNJ1, P19096; CFC $=1.69$ ) indicate the presence of glycolysis and proxy-citrate cycle derived fatty acid synthase I (FAS I) system in the jejunum and the ileum, as reported previously [41].

Compared to the small intestine, glutamine/glutamate metabolism was upregulated in the infected caecum. Glucose depletion in the caecum and the colon is known to trigger glutamate utilisation as the primary carbon source, by both Cryptosporidium and host defence cells [6, 42]. In the context of cryptosporidiosis, we observed glutamate utilisation typical of parasitic activity for generating aketoglutarate, catalysed by glutamine synthetase, glutamate kinase, and glutamate-5-semialdehyde dehydrogenase, as previously documented [6].

We observed upregulated host and yeast transketolases, followed by yeast polyubiquitin proteins, indicative of these proteins/enzymes catalysing ubiquinone biosynthesis in the jejunum-ileum tract. The preliminary step of the ubiquinone biosynthesis pathway begins with E4P metabolism and is catalysed by glucose-6-phosphate dehydrogenase (G6PDH) in trypanosomatid [43] and Plasmodium [44] metabolism, and during $C$. parvum infection in rats [45]. The utilisation of enzyme systems from the host, yeasts, and the parasite indicates a host-parasite-microbiome association in the small intestine. This association may have compensated the deficient Cryptosporidium metabolic machinery for synthesising ubiquinone (coenzyme Q), which is a critical element of the electron transport chain. Such associations and their benefits to Cryptosporidium multiplication have been reported in aquatic systems [17] and neonatal mice gut dysbiosis [18].

High yeast ubiquitin-related activity was observed across the small intestine, especially in the ileum. While 11 mouse ubiquitin transfer or conjugating enzymes were observed to be upregulated throughout the host response $(F C=1.1-10.4)$, yeast ubiquitin/polyubiquitin proteins were also upregulated $(F C=$ 
1107 - 1960). These proteins are required for synthesis, transfer, and metabolism of ubiquinone. While it has been reported that Cryptosporidium salvages the host ubiquinone system [6], our study indicated significant salvaging of this system from the yeast population of the microbiome. To our knowledge, the current study is the first to suggest that enhanced Cryptosporidium colonisation may depend more on the parasite-microbiome relationship than the host-parasite relationship.

\section{Extra-intestinal effects of cryptosporidiosis}

In non-gut organs such as the liver, oxalic acid upregulation is indicative of likely hyperoxaluria or a hyperoxaluria-like condition. In this condition, glyoxylate metabolism is negatively affected due to the deficiency of hepatic alanine glyoxylate aminotransferase (AGT) and cytosolic glyoxylate reductase (GR) $[46,47]$. However, the relationship to hyperoxaluria as an indirect effect of Cryptosporidium infection in the gut remains to be determined.

Mitochondrial pyruvate carboxylase was possibly one of the most interesting of the expressed proteins in the liver. This zinc-containing protein, in the presence of allosteric activators such as acetyl-CoA, catalyses the pyruvate " oxaloacetate reaction towards both Krebs cycle replenishment and gluconeogenesis [48]. However, an excessive accumulation of oxalate (caused by oxaloacetate accumulation) in the liver of Cryptosporidium-infected mice may be attributed to the high expression of Llactate dehydrogenase (LDH). The role of hepatic LDH in converting glyoxylate to oxalate has recently been reported for primary hyperoxaluria mouse models [49] and blood-based protozoal infections such as that with Plasmodium [50]. However, its indirect hepatic activity, especially as a follow-up pyruvate carboxylase activity, due to gut infection has not been reported and its dynamics require further study.

\section{Conclusions}

We utilised a mouse model to study the direct (gut) and indirect (serum and liver) effects of cryptosporidiosis using a multi-omics approach. Energy pathways such as glycolysis and glutaminolysis were significantly impacted in the jejunum and ileum during cryptosporidiosis. The proteomic and metabolic outputs indicated an underdeveloped proxy-citrate cycle in Cryptosporidium, partially salvaged from the host, with additional input of yeast citrate cycle enzymes. Instead of the commonly reported G6PDH-catalysed route, the ubiquinone (CoQ) biosynthesis system in the ileum began with host transketolase activity, followed by the salvation of the yeast ubiquinone biosynthetic system. The gut microbiome response to cryptosporidiosis was detected via increased metabolism of D-amino acids and SCFAs. Similarly, high oxalate accumulation in the liver indicated enteric hyperoxaluria as a likely indirect effect of cryptosporidiosis. Our study shows the ability of multi-omics to contribute a robust understanding of gut infections and demonstrate the previously unreported microbiome interaction dynamics during cryptosporidiosis. The study highlights the comprehensive dynamics of gut infection and may serve as a model that can be used for the in-depth study of other enteric infections. These results provide a platform from which new avenues of precision medicine and improved treatment methods for cryptosporidiosis may be devised. 


\section{Declarations}

\section{Ethics approval and consent to participate}

All experiments were approved by the Monash University Animal Ethics Committee (Monash University AEC no. MARP/2018/055) following the guidelines of Victorian State Government and The National Health and Medical Research Council, Australian Government.

\section{Consent for publication}

Not applicable

\section{AVAILABILITY OF DATA AND MATERIALS}

Please contact senior authors (DJB, DL and EAP) for data requests.

\section{Competing interests/Conflict of interest statement}

The authors declare no conflict of interest.

\section{Funding}

This work was completed as a part of the CSIRO Early Research Career (CERC) postdoctoral fellowship program with additional support provided by the CSIRO Probing Biosystems Future Science Platform (FSP).

\section{Author contributions to manuscript}

AVK, DJB, MLH, DL, EAP: Concept, experiment design, planning, analysis, manuscript drafting, and critical review. RMS, KEH, SJM: Data analysis, interpretation, manuscript drafting, and critical review. SSM, ABG, TW: genomic analysis, manuscript drafting. J-WL: Proteomic sample process and analysis. SJM, MJ, CE: Mouse experiments and animal handling

\section{Acknowledgments}

Graphical abstract and Figure 1 were created with BioRender online visualisation tool (biorender.com). The authors wold like to acknowledge the help provided by Professor Stephen Rose to provide the operational costs to the study through the CSIRO Probing Biosystems Future Science Platform (FSP).

\section{References}

1. Berger S: Cryptosporidiosis: Global Status. Los Angeles, CA, UNITED STATES: GIDEON Informatics Inc; 2017.

2. Certad G, Viscogliosi E, Chabé M, Cacciò SM: Pathogenic mechanisms of Cryptosporidium and Giardia. Trends in Parasitology 2017, 33(7):561-576. 
3. Chalmers RM: Chapter Sixteen - Cryptosporidium. In: Microbiology of Waterborne Diseases (Second Edition). London: Academic Press; 2014: 287-326.

4. Priyamvada S, Kumar A, Anbazhagan AN, Jayawardena D, Alrefai WA, Dudeja PK, Bortahkur A: Cryptosporidium parvum infection induces autophagy in intestinal epithelial cells. Gastroenterology 2019, 156(6):S-668.

5. Alcock F, Webb CT, Dolezal P, Hewitt V, Shingu-Vasquez M, Likić VA, Traven A, Lithgow T: A small tim homohexamer in the relict mitochondrion of Cryptosporidium. Molecular Biology and Evolution 2011, 29(1):113-122.

6. Shanmugasundram A, Gonzalez-Galarza FF, Wastling JM, Vasieva O, Jones AR: Library of apicomplexan metabolic pathways: a manually curated database for metabolic pathways of apicomplexan parasites. Nucleic acids research 2012, 41(D1):D706-D713.

7. Yu Y, Zhang H, Guo F, Sun M, Zhu G: A unique hexokinase in Cryptosporidium parvum, an apicomplexan pathogen lacking the Krebs cycle and oxidative phosphorylation. Protist 2014, 165(5):701-714.

8. Stiff RE, Davies AP, Mason BW, Hutchings HA, Chalmers RM: Long-term health effects after resolution of acute Cryptosporidium parvum infection: a 1-year follow-up of outbreak-associated cases. Journal of Medical Microbiology 2017, 66(11):1607-1611.

9. Bi H, Krausz K, Manna S, Li F, Johnson C, Gonzalez F: Optimization of harvesting, extraction, and analytical protocols for UPLC-ESI-MS-based metabolomic analysis of adherent mammalian cancer cells. Anal Bioanal Chem 2013, 405(15):5279-5289.

10. Marcinowska R, Trygg J, Wolf-Watz H, Mortiz T, Surowiec I: Optimization of a sample preparation method for the metabolomic analysis of clinically relevant bacteria. J Microbiol Meth 2011, 87(1):2431.

11. Niklas J, Schneider K, Heinzle E: Metabolic flux analysis in eukaryotes. Curr Opin Biotech 2010, 21(1):63-69.

12. Beale DJ, Morrison PD, Karpe AV, Dunn MS: Chemometric analysis of lavender essential oils using targeted and untargeted GC-MS acquired data for the rapid identification and characterization of oil quality. Molecules 2017, 22(8):1339.

13. Beale D, Karpe A, Ahmed W, Cook S, Morrison P, Staley C, Sadowsky M, Palombo E: A community multi-omics approach towards the assessment of surface water quality in an urban river system. International Journal of Environmental Research and Public Health 2017, 14(3):303.

14. Kumarasingha R, Karpe AV, Preston S, Yeo T-C, Lim DSL, Tu C-L, Luu J, Simpson KJ, Shaw JM, Gasser RB et al: Metabolic profiling and in vitro assessment of anthelmintic fractions of Picria felterrae Lour. International Journal for Parasitology: Drugs and Drug Resistance 2016, 6(3):171-178.

15. Robinson AM, Gondalia SV, Karpe AV, Eri R, Beale DJ, Morrison PD, Palombo EA, Nurgali K: Fecal microbiota and metabolome in a mouse model of spontaneous chronic colitis: Relevance to human inflammatory bowel disease. Inflammatory Bowel Diseases 2016, 22(12):2767-2787. 
16. Beale DJ, Marney D, Marlow DR, Morrison PD, Dunn MS, Key C, Palombo EA: Metabolomic analysis of Cryptosporidium parvum oocysts in water: A proof of concept demonstration. Environ Pollut 2013, 174(Complete):201-203.

17. Koh W, Clode PL, Monis P, Thompson RA: Multiplication of the waterborne pathogen Cryptosporidium parvum in an aquatic biofilm system. Parasites \& Vectors 2013, 6(1):270.

18. Mammeri M, Chevillot A, Thomas M, Julien C, Auclair E, Pollet T, Polack B, Vallée I, Adjou KT: Cryptosporidium parvum-infected neonatal mice show gut microbiota remodelling using highthroughput sequencing analysis: Preliminary results. Acta Parasitologica 2019, 64(2):268-275.

19. Ng JSY, Ryan U, Trengove RD, Maker GL: Development of an untargeted metabolomics method for the analysis of human faecal samples using Cryptosporidium-infected samples. Mol Biochem Parasit 2012, 185(2):145-150.

20. Sarkar S, Hutton ML, Vagenas D, Ruter R, Schüller S, Lyras D, Schembri MA, Totsika M: Intestinal colonization traits of pandemic multidrug-resistant Escherichia coli ST131. The Journal of Infectious Diseases 2018, 218(6):979-990.

21. Nielsen KL, Dynesen P, Larsen P, Frimodt-Møller N: Faecal Escherichia coli from patients with E. coli urinary tract infection and healthy controls who have never had a urinary tract infection. Journal of medical microbiology 2014, 63(4):582-589.

22. Fiehn O: Metabolomics by gas chromatography-mass spectrometry: Combined targeted and untargeted profiling. Current protocols in molecular biology 2016, 114(1):30.34. 31-30.34. 32.

23. Beale DJ, Oh DY, Karpe AV, Tai C, Dunn MS, Tilmanis D, Palombo EA, Hurt AC: Untargeted metabolomics analysis of the upper respiratory tract of ferrets following influenza $A$ virus infection and oseltamivir treatment. Metabolomics 2019, 15(3):33.

24. Beale D, Karpe A, Rupprecht U: In-time TMS derivatization and GC/MS determination of sugars, organic acids and amino acids for high throughput metabolomics studies. In: GERSTEL application notes. 2020: 1-10.

25. Bolyen E, Rideout JR, Dillon MR, Bokulich NA, Abnet CC, Al-Ghalith GA, Alexander H, Alm EJ, Arumugam M, Asnicar $\mathrm{F}$ et al: Reproducible, interactive, scalable and extensible microbiome data science using QIIME 2. Nature biotechnology 2019, 37(8):852-857.

26. Arndt D, Xia J, Liu Y, Zhou Y, Guo AC, Cruz JA, Sinelnikov I, Budwill K, Nesbø CL, Wishart DS: METAGENassist: a comprehensive web server for comparative metagenomics. Nucleic acids research 2012, 40(W1):W88-W95.

27. Chong J, Soufan O, Li C, Caraus I, Li S, Bourque G, Wishart DS, Xia J: MetaboAnalyst 4.0: towards more transparent and integrative metabolomics analysis. Nucleic acids research 2018, 46(W1):W486-W494.

28. Hernández-de-Diego R, Tarazona S, Martínez-Mira C, Balzano-Nogueira L, Furió-Tarí P, Pappas GJ, Jr., Conesa A: PaintOmics 3: a web resource for the pathway analysis and visualization of multi-omics data. Nucleic acids research 2018, 46(W1):W503-w509. 
29. Vemuri R, Gundamaraju R, Shinde T, Perera AP, Basheer W, Southam B, Gondalia SV, Karpe AV, Beale DJ, Tristram S: Lactobacillus acidophilus DDS-1 modulates intestinal-specific microbiota, short-chain fatty acid and immunological profiles in aging mice. Nutrients 2019, 11(6):1297.

30. Sasabe J, Miyoshi Y, Rakoff-Nahoum S, Zhang T, Mita M, Davis BM, Hamase K, Waldor MK: Interplay between microbial D-amino acids and host D-amino acid oxidase modifies murine mucosal defence and gut microbiota. Nature Microbiology 2016, 1(10):16125.

31. Ras R, Huynh K, Desoky E, Badawy A, Widmer G: Perturbation of the intestinal microbiota of mice infected with Cryptosporidium parvum. International Journal for Parasitology 2015, 45(8):567-573.

32. Roychowdhury S, Cadnum J, Glueck B, Obrenovich M, Donskey C, Cresci GAM: Faecalibacterium prausnitzii and a prebiotic protect intestinal health in a mouse model of antibiotic and Clostridium difficile exposure. Journal of Parenteral and Enteral Nutrition 2018, 42(7):1156-1167.

33. Rowin J, Xia Y, Jung B, Sun J: Gut inflammation and dysbiosis in human motor neuron disease. Physiological Reports 2017, 5(18):e13443.

34. De Maesschalck C, Van Immerseel F, Eeckhaut V, De Baere S, Cnockaert M, Croubels S, Haesebrouck F, Ducatelle R, Vandamme P: Faecalicoccus acidiformans gen. nov., sp. nov., isolated from the chicken caecum, and reclassification of Streptococcus pleomorphus (Barnes et al. 1977), Eubacterium biforme (Eggerth 1935) and Eubacterium cylindroides (Cato et al. 1974) as Faecalicoccus pleomorphus comb. nov., Holdemanella biformis gen. nov., comb. nov. and Faecalitalea cylindroides gen. nov., comb. nov., respectively, within the family Erysipelotrichaceae. International journal of systematic and evolutionary microbiology 2014, 64(11):3877-3884.

35. Chang D-H, Rhee M-S, Ahn S, Bang B-H, Oh JE, Lee HK, Kim B-C: Faecalibaculum rodentium gen. nov., sp. nov., isolated from the faeces of a laboratory mouse. Antonie van Leeuwenhoek 2015, 108(6):1309-1318.

36. Shao DY, Bai X, Tong MW, Zhang Yy, Liu XI, Zhou Yh, Li C, Cai W, Gao X, Liu M et al: Changes to the gut microbiota in mice induced by infection with Toxoplasma gondii. Acta Tropica 2020, 203:105301.

37. Liu H, Zhang H, Wang X, Yu X, Hu C, Zhang X: The family Coriobacteriaceae is a potential contributor to the beneficial effects of RouX-en-Y gastric bypass on type 2 diabetes. Surgery for Obesity and Related Diseases 2018, 14(5):584-593.

38. Kobayashi J: d-Amino acids and lactic acid bacteria. Microorganisms 2019, 7(12):690.

39. Rosa LT, Bianconi ME, Thomas GH, Kelly DJ: Tripartite ATP-independent periplasmic (TRAP) transporters and tripartite tricarboxylate transporters (TTT): From uptake to pathogenicity. Frontiers in Cellular and Infection Microbiology 2018, 8(33).

40. Xu Z, Guo Y, Roellig DM, Feng Y, Xiao L: Comparative analysis reveals conservation in genome organization among intestinal Cryptosporidium species and sequence divergence in potential secreted pathogenesis determinants among major human-infecting species. BMC Genomics 2019 , 20(1):406. 
41. Guo F, Zhang H, Fritzler JM, Rider SD, Jr, Xiang L, McNair NN, Mead JR, Zhu G: Amelioration of Cryptosporidium parvum infection in vitro and in vivo by targeting parasite fatty acyl-coenzyme $A$ synthetases. The Journal of Infectious Diseases 2013, 209(8):1279-1287.

42. Wang R, Wang T, Tait S: Chapter $\mathbf{2 0}$ - Metabolic regulation of immunity. In: Kelley and Firestein's Textbook of Rheumatology (Tenth Edition). Edited by Firestein GS, Budd RC, Gabriel SE, McInnes IB, O'Dell JR: Elsevier; 2017: 318-326.

43. Gupta S, Igoillo-Esteve M, Michels PAM, Cordeiro AT: Glucose-6-Phosphate dehydrogenase of trypanosomatids: Characterization, target validation, and drug discovery. Molecular Biology International 2011, 2011.

44. Choudhary HH, Srivastava PN, Singh S, Kumar KA, Mishra S: The shikimate pathway enzyme that generates chorismate is not required for the development of Plasmodium berghei in the mammalian host nor the mosquito vector. International Journal for Parasitology 2018, 48(3):203-209.

45. Rasmussen KR, Arrowood MJ, Healey MC: Effectiveness of dehydroepiandrosterone in reduction of cryptosporidial activity in immunosuppressed rats. Antimicrobial agents and chemotherapy 1992, 36(1):220-222.

46. Langlois V: CHAPTER 2 - Laboratory evaluation at different ages. In: Comprehensive Pediatric Nephrology. Edited by Geary DF, Schaefer F. Philadelphia: Mosby; 2008: 39-54.

47. Matson E, Reginato AM: Chapter 23 - Crystalline disorders associated with renal disease including oxalate arthropathy. In: Gout \& Other Crystal Arthropathies. Edited by Terkeltaub R. Philadelphia: W.B. Saunders; 2012: 282-292.

48. Engelking LR: Chapter 27 - Metabolic fates of pyruvate. In: Textbook of Veterinary Physiological Chemistry (Third Edition). Edited by Engelking LR. Boston: Academic Press; 2015: 169-173.

49. Lai C, Pursell N, Gierut J, Saxena U, Zhou W, Dills M, Diwanji R, Dutta C, Koser M, Nazef N et al: Specific inhibition of hepatic lactate dehydrogenase reduces oxalate production in mouse models of primary hyperoxaluria. Molecular Therapy 2018, 26(8):1983-1995.

50. Wirth JD, Boucher JI, Jacobowitz JR, Classen S, Theobald DL: Functional and structural resilience of the active site loop in the evolution of Plasmodium lactate dehydrogenase. Biochemistry 2018, 57(45):6434-6442.

\section{Figures}




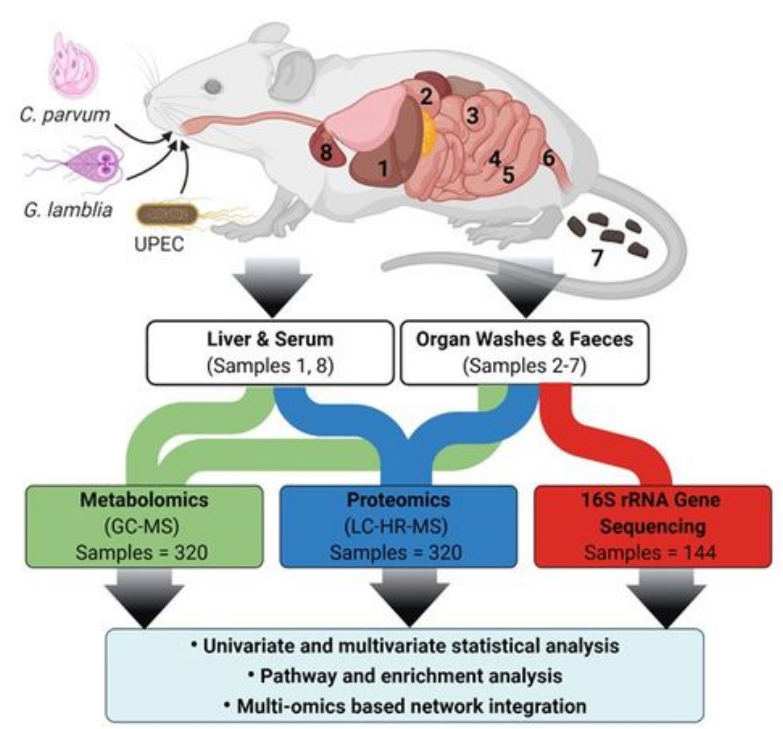

Figure 1

\section{Figure 1}

Overview of mouse cryptosporidiosis interaction study design showing various mouse samples that were collected. Samples were subjected to GC-MS and LC-HR-MS and resulting data were analysed by multivariate statistics. Samples are annotated as (1) liver tissue, washes of (2) duodenum, (3) jejunum, (4) ileum, (5) caecum, (6) colon, (7) faeces, and (8) serum.

(A)

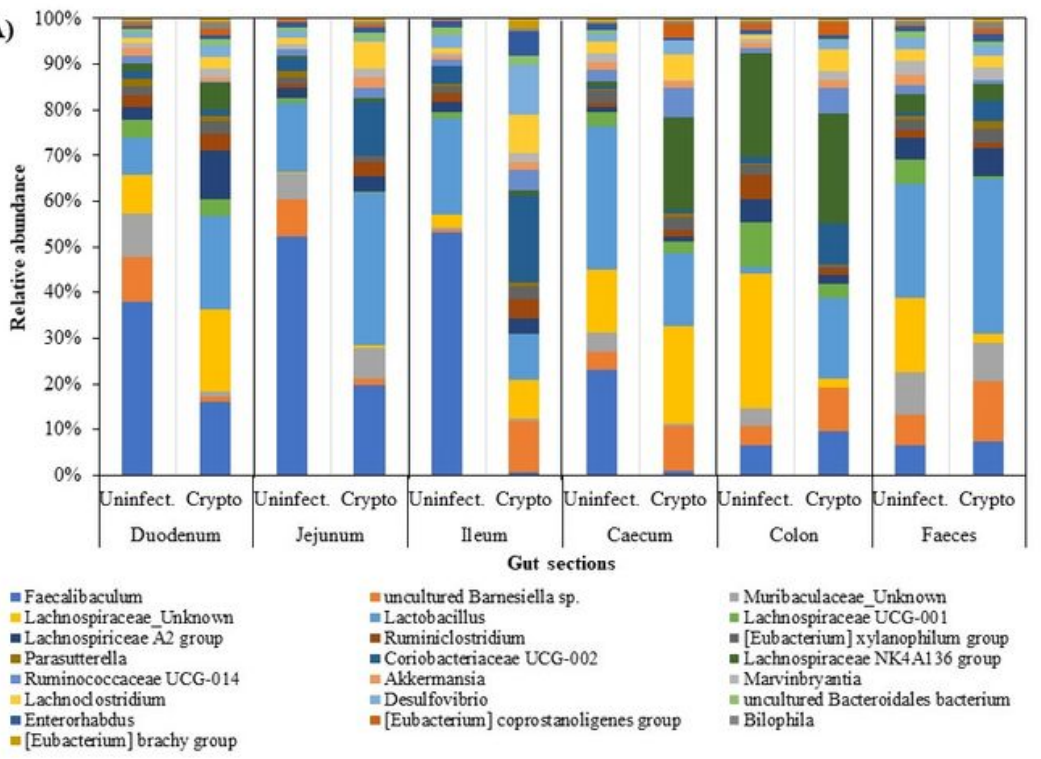

(B)

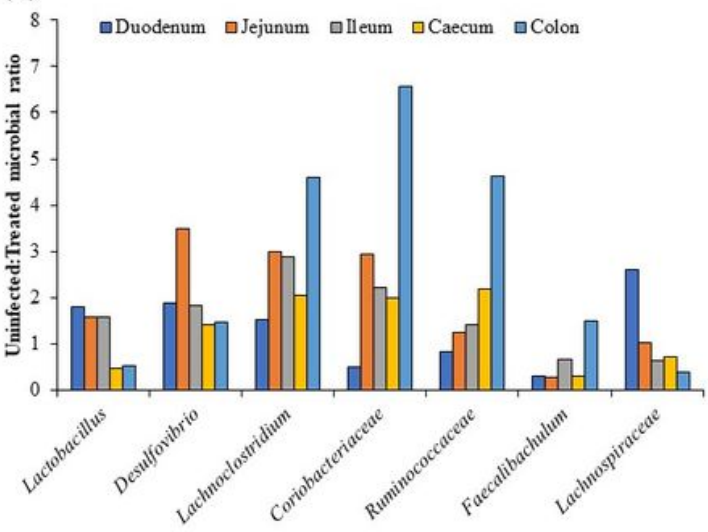

Figure 2

\section{Figure 2}


The relative abundance of $(A)$ major/core genera across regions of the intestinal system of uninfected and Cryptosporidium-infected mice and, (B) ratio of major genera showing prominent changes during cryptosporidiosis infection compared to the uninfected mice, in mouse intestine regions.

(A)

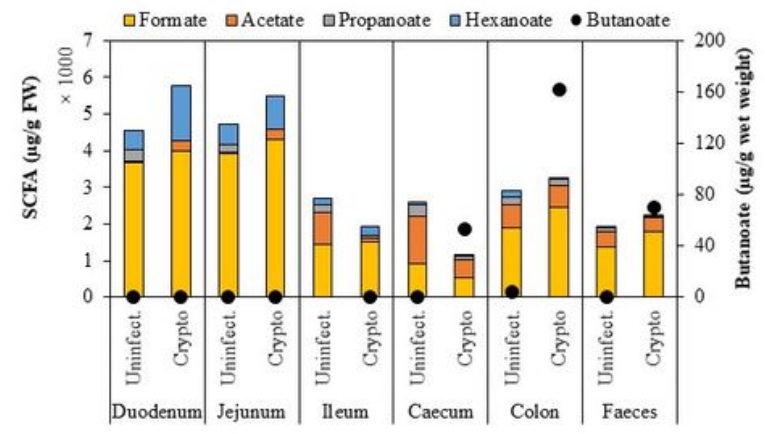

(B)

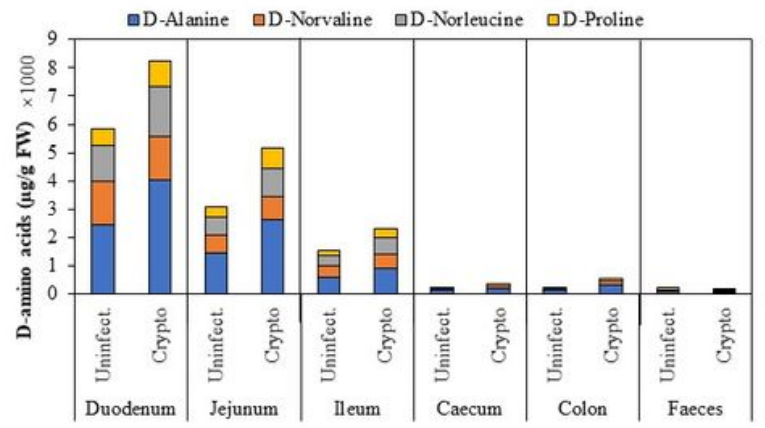

Figure 3

\section{Figure 3}

Distribution of (A) Short chain fatty acids (SCFAs) and (B) D-amino acids across various regions of the intestinal tract ( $\mu \mathrm{g} / \mathrm{g}$ FW of samples) of uninfected and Cryptosporidium-infected mice. Note: Individual charts are presented in Figures S5 and S6.

(A)

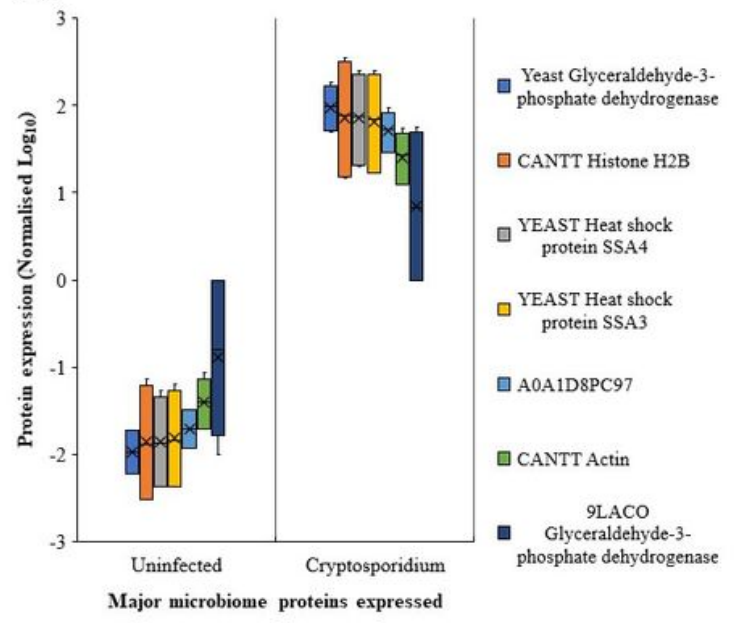

(B)

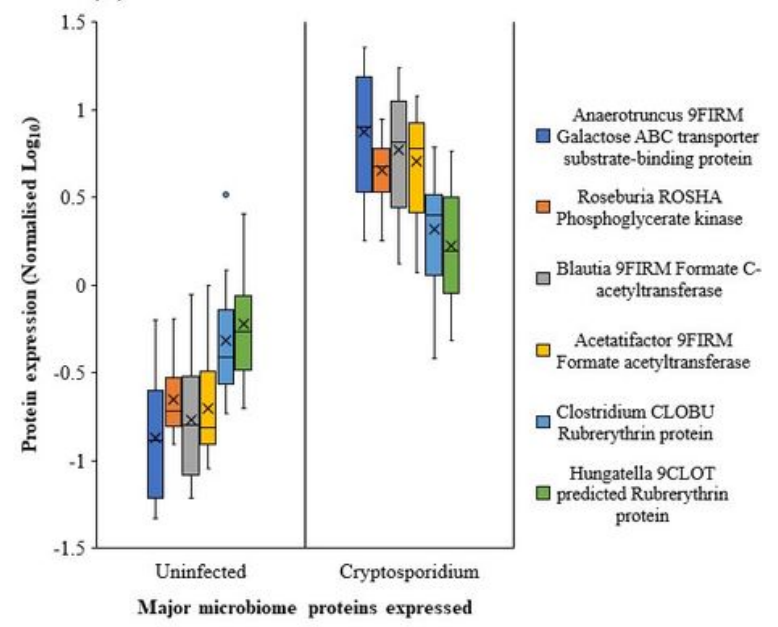

Figure 4

Figure 4 
Major proteins expressed by microbial community in response to cryptosporidiosis in (A) the jejunumileum of the small intestine and (B) the caecum-colon and faeces.

(A)

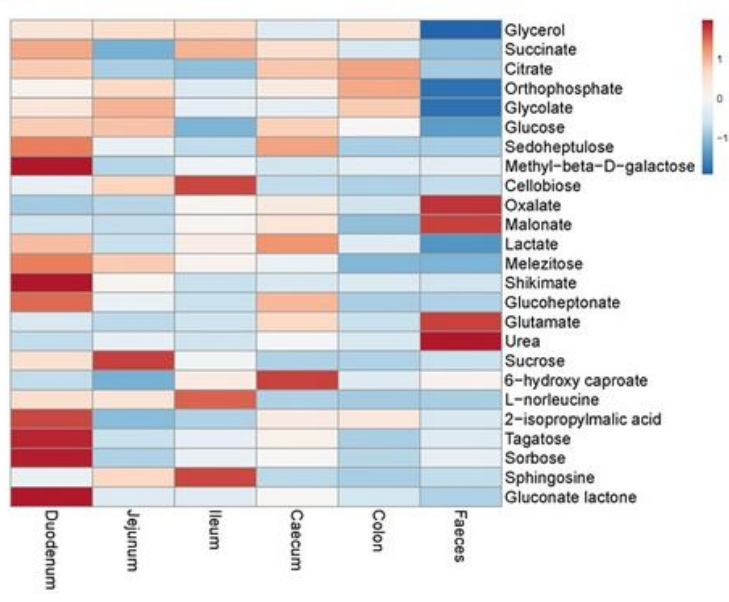

(B)

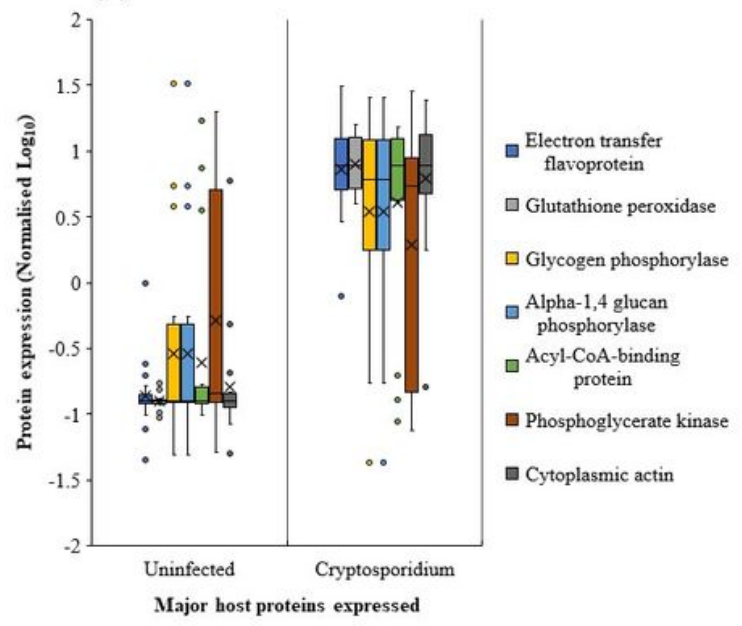

Figure 5

\section{Figure 5}

(A) List of top 25 metabolites (in descending order) with significantly high variable importance in projection (VIP) scores in the mouse gut during Cryptosporidium infection (Refer to Table S13 for data). (B) Most prominent host proteins expressed across the mouse intestine upon Cryptosporidium infection, the citrate cycle and oxidative phosphorylation.

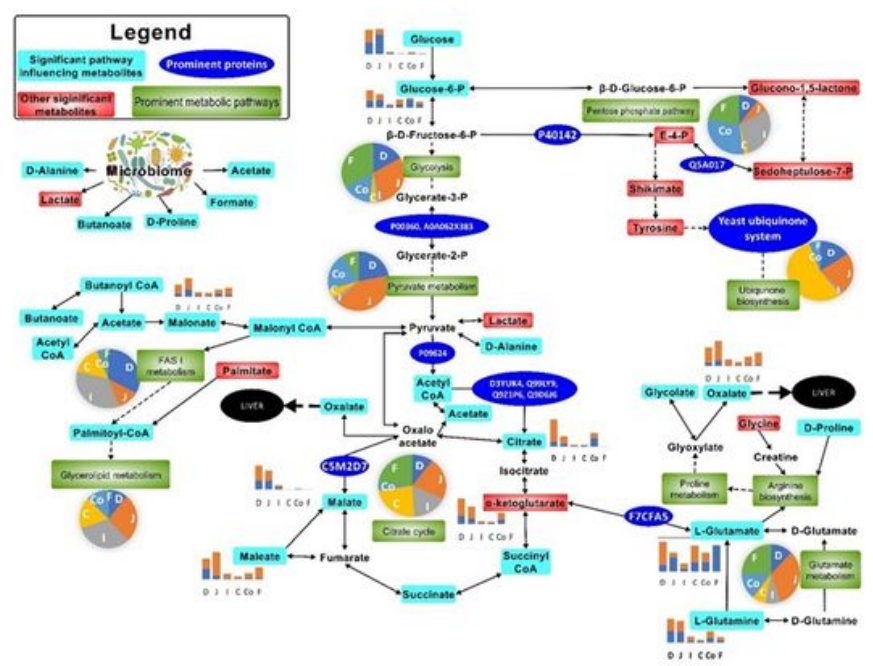

Figure 6 
Most prominent metabolic activities in the mouse gut upon Cryptosporidium infection. The pie charts indicate the relative impact of pathways in the duodenum (D), jejunum (J), ileum (I), caecum (C), colon (Co), and faeces (F). The bar graphs show perturbed metabolites in uninfected and Cryptosporidium infected mice. Note: Please refer to Figure $\mathrm{S} 8$ for a more descriptive pathway chart.

\section{Supplementary Files}

This is a list of supplementary files associated with this preprint. Click to download.

- Graphicalabstract.JPG

- SD1CryptosporidiosisMicrobiomeresponselntestine.xlsx

- SD2Cryptosporidiosishostresponselntestine.xlsx

- SD3CryptosporidiosishostresponseSerumLiver.xlsx

- SupplementaryCryptosporidiosisMicrobiome.docx 